\title{
LETTER
}

\section{Angiogenic factors in sepsis: are we ready for the new therapeutic era?}

\author{
Ru-Yuan Zhang, Hong Zhang, Jie Huang, Hong-Ping Qu and Yao-Qing Tang* \\ See related research by Fiusa et al., http://ccforum.com/content/17/4/R169
}

Recently, Fiusa and colleagues [1] described that a high angiopoietin (Ang)-2/Ang-1 ratio is associated with a high risk of septic shock in patients with febrile neutropenia. Although this study failed to detect a statistically significant difference in the levels of vascular endothelial growth factor (VEGF) between both outcome groups, the observed increased Ang-2 concentrations and Ang-2/Ang-1 ratio in the febrile neutropenia subgroup of patients with septic shock address the concept that angiogenic factors could be a promising therapeutic target in sepsis.

The imbalance of angiogenic factors and their receptors, including the Ang/Tie2 and VEGF/VEGF receptor pathways, has been shown to predict poor prognosis in sepsis and has been implicated as a contributing factor in multiple organ dysfunction. For these reasons, several strategies targeting Ang/Tie2 and VEGF/VEGF receptor are being explored in preclinical sepsis models; however, most of these agents are unavailable or not applicable in clinical practice. Efforts to develop anticancer agents that target angiogenesis have led to the approval of inhibitors of VEGF and its receptor, specifically bevacizumab. As recently reported in Critical Care, bevacizumab has proven effective in the treatment of sepsis in an animal model [2].

The current study reinforces and extends previous findings that Ang-2 levels are increased in the septic state and have been established as a prognostic biomarker for identification of the patient group with the highest mortality rates. Interestingly, most recently by using Ang-2 antibody treatment, Ziegler and colleagues [3] showed that inhibition of Ang-2 has a significant effect on survival of mice with sepsis. AMG 386 is an investigational peptide-Fc fusion protein that inhibits angiogenesis by preventing the interaction of

\footnotetext{
* Correspondence: yaoqing.tang@hotmail.com

Department of Critical Care Medicine, Rui Jin Hospital, Shanghai Jiao Tong University School of Medicine, 197 Rui Jin 2nd Road, Shanghai 200025, China
}

Ang-1/2 with their receptor, Tie2. The human study demonstrated that intravenous AMG 386 appeared well tolerated, and its safety profile seemed to be distinct from that of VEGF axis inhibitors [4]; it has reached late stages of clinical development. This provides a basis for Ang-2 inhibition as a potential clinical-translational therapeutic target in sepsis. The distinct safety profiles of AMG 386 and the VEGF-axis inhibitors suggest that they could be combined to achieve cooperative action, which may thereby improve clinical efficacy without significant overlapping toxicities.

Thus, recent findings indicate that angiogenic factors are potential therapeutic targets in sepsis. However, the safety profiles of these anti-angiogenic agents in septic patients are often complicated by organ dysfunction and may be different from those in cancer patients. Future clinical trials may be needed to evaluate the safety and efficacy of these anti-angiogenic agents in sepsis patients, either alone or in combination.

\section{Abbreviations \\ Ang: Angiopoietin; VEGF: Vascular endothelial growth factor.}

\section{Competing interests}

The authors declare that they have no competing interests.

\section{Authors' contributions}

RYZ, YQT and HPQ developed the concept and drafted the manuscript. $\mathrm{HZ}$ and $\mathrm{JH}$ searched the literature and analyzed data. All authors read and approved the final draft of the manuscript.

\section{Acknowledgements}

This work was supported by the National Natural Science Foundation of China (NSFC, grant number 81071534).

\section{Published: 29 Jan 2014}

\section{References}

1. Fiusa MM, Costa-Lima C, de Souza GR, Vigorito AC, Aranha FJ, Lorand-Metze I, Annichino-Bizzacchi JM, De Souza CA, De Paula EV: A high angiopoietin-2/ angiopoietin-1 ratio is associated with a high risk of septic shock in patients with febrile neutropenia. Crit Care 2013, 17:R169. 
2. Jeong SJ, Han SH, Kim CO, Choi JY, Kim JM: Anti-vascular endothelial growth factor antibody attenuates inflammation and decreases mortality in an experimental model of severe sepsis. Crit Care 2013, 17:R97.

3. Ziegler T, Horstkotte J, Schwab C, Pfetsch V, Weinmann K, Dietzel S, Rohwedder I, Hinkel R, Gross L, Lee S, Hu J, Soehnlein O, Franz WM, Sperandio M, Pohl U, Thomas M, Weber C, Augustin HG, Fassler R, Deutsch U, Kupatt C: Angiopoietin 2 mediates microvascular and hemodynamic alterations in sepsis. J Clin Invest 2013.

4. Herbst RS, Hong D, Chap L, Kurzrock R, Jackson E, Silverman JM, Rasmussen E, Sun YN, Zhong D, Hwang YC, Evelhoch JL, Oliner JD, Le N, Rosen LS:

Safety, pharmacokinetics, and antitumor activity of AMG 386, a selective angiopoietin inhibitor, in adult patients with advanced solid tumors. J Clin Oncol 2009, 27:3557-3565.

\section{$10.1186 /$ cc13710}

Cite this article as: Zhang et al:: Angiogenic factors in sepsis: are we ready for the new therapeutic era? Critical Care 2014, 18:403 